\title{
OBSERVED HAND WASHING PRACTICES AMONG HEALTH WORKERS IN TWO CRITICAL PAEDIATRICS WARDS OF A SPECIALIST HOSPITAL
}

\author{
Balafama Abinye Alex-Hart and Peace Ibo Opara \\ Department of Paediatrics, University of Port Harcourt Teaching Hospital, Port Harcourt, Nigeria
}

Received 2014-05-26; Revised 2014-07-07; Accepted 2014-07-11

\begin{abstract}
Hand washing in between patient care by health workers is the single most important measure against occurrence and spread of nosocomial infections within health facilities. This study was done to observe health workers hand washing practices in two critical Paediatric wards of a specialist hospital. Trained observers observed and recorded health workers' hand washing compliance while carrying out their routine patient care. Other information recorded included the time of observation and health workers' occupation and rank. Data was fed in to excel spread sheet and analyzed using SPSS version 16.0. A total of 150 health workers were observed in this study. There were $116(77.3 \%)$ females and $34(22.7 \%)$ males giving a male: Female ratio of 1: 3.4. There were $86(57.3 \%)$ doctors and $64(42.7 \%)$ nurses. During the period of observation, soap with running water was found in only $39(26.0 \%)$ occasions. Common cotton towel was found in $78.7 \%$ of the period of observation as the only available hand drying facility. Doctors' hand washing rates before and after patients contact were 17.4 and $64.0 \%$ respectively. Doctors' hand washing rates before and after simple procedures ranged from 0 to 56.5 and 60.6 to $100 \%$ respectively. Nurses' hand washing rates before and after simple procedures ranged from 1.3 to $28.6 \%$ and 19.7 to $88.4 \%$ respectively. Health workers (doctors and nurses) hand washing rates on entering the wards was $4 \%$. Hand washing rate before leaving the wards was $74.7 \%$. Majority of the health workers dried their hands with non-disposable common cotton towels on $72.0 \%$ of the occasions. Hand washing rates was very low before patient contact and before simple procedures.
\end{abstract}

Keywords: Observed, Hand Washing Practices, Health Workers, Critical Paediatric Wards, Nosocomial Infections

\section{INTRODUCTION}

Nosocomial infections are a global problem affecting more than 1.4 million patients at any time worldwide. WHO (2005). In developed countries it affects 5-15\% of all hospitalized patients and $9-37 \%$ of those admitted into intensive care units (WHO, 2005; Vincent, 2003). In developing countries, prevalence rates of 19.1, 17.9, 17.8 and $14.8 \%$ have been reported in Abania, Tunisia, Morocco and United Republic of Tanzania respectively (Faria et al., 2007; Kallel et al., 2005; Jroundi et al., 2007; Gosling et al., 2003). Most of these infections result from transmission of infectious agents from one patient to the other via the hands of health workers. Hand washing with soap and water by health workers at appropriate times is therefore the singular most effective preventive measure against the spread of these healthcare associated infections. Against this background, the World Health Organization (WHO) formulated guidelines on hand hygiene with the ultimate objective of changing the behaviour of individual health workers to optimize compliance with hand hygiene at the recommended moments to improve patient safety. WHO (2009). Nevertheless, despite the simplicity of hand Corresponding Author: Balafama Abinye Alex-Hart, Department of Paediatrics, University of Port Harcourt Teaching Hospital, Port Harcourt, Nigeria Tel: +234 8033091084 
washing procedures, hand washing rates amongst health workers is still reported as very low (Pittet et al., 2004; Lankford et al., 2003). Some authors have blamed the low hand washing rates on poor compliance with the hand hygiene guidelines by health workers (Larson, 1995; Pittet et al., 1999).

Studies have shown that when health workers comply with the hand washing policies within health facilities, there is reduction in the frequency and severity of hospital associated infections (Akpaka, 2014; Larson, 1995; Pittet, 2003). A previous study done in Nigeria on hand washing compliance by health workers showed low rates, although it was a self-reported study. Alex-Hart and Opara (2011). Currently, observational surveys conducted by trained personnel are considered the gold standard for monitoring hand hygiene compliance among health care workers (Pritchard et al., 1996; Boyce, 2008). This is because observational studies have the ability to determine if hand hygiene is being performed at the correct times. Furthermore it can establish compliance rates by health care workers and assess hand hygiene techniques. Boyce (2008).

Very ill children are usually managed in children's emergency wards, whilst very ill term and preterm neonates are managed in the neonatal intensive care unit. There is therefore a high risk of transmission of nosocomial infections from patient to patient in these critical sections of Paediatric care. Good hand washing practices are therefore very important in such wards. This study was carried out to observe health workers hand washing practices in the Children's emergency and the neonatal intensive care wards.

\section{MATERIALS AND METHODS}

This was a cross sectional observational study carried out in children's emergency and neonatal intensive care wards of a tertiary hospital in Port Harcourt over a 30day period. Two doctors were trained on observing health care workers' (HCWs) hand washing practices during hand washing opportunities. Data collection sheets were prepared for recording observations for each health worker being observed. Doctors and nurses working in children's emergency and neonatal intensive care wards were observed during routine patient care for compliance with hand washing. The observing doctors unobtrusively recorded health workers hand washing practices in the data sheets. The opportunities for hand washing included those opportunities where the HCW did invasive procedures, came into personal contact with patients or patients' body fluids, or did non-invasive procedures such as blood pressure or temperature measurement or intermittent positive pressure ventilation. Hand washing was defined as washing hands with soap and running water. No waterless alternative was available for other types of hand hygiene throughout the period of observation. The observation periods were distributed randomly during the day as well as at night for 30 days. The subjects were unaware that they were being observed. Each subject was observed once and the observation was recorded with the time of the event. The HCWs occupation and rank were also recorded.

\section{RESULTS}

A total of $150 \mathrm{HCW}$ sere observed in this study. There were $116(77.3 \%)$ females and 34 (22.7\%) males giving a male: Female ratio of 1: 3.4. There were 86 $(57.3 \%)$ doctors and $64(42.7 \%)$ nurses. Out of the 86 doctors, $45(52.3 \%)$ were registrars, 24 (27.9\%) were house officers, $11(12.8 \%)$ were senior registrars and 6 $(7.0 \%)$ were consultants. Of the 64 nurses, 27 (42.2\%) were matrons, $19(29.7 \%)$ were staff nurses and 18 (28.1\%) were senior nursing officers.

Fifty six $(37.3 \%)$ observations occurred during the night shift, $52(34.7 \%)$ during the morning shift and 42 (28\%) during the afternoon shift. During the period of observation, soap with running water was found in 39 (26\%) occasions, running water alone was found in 110 $(73.3 \%)$ and on $1(0.7 \%)$ occasion, no facility for hand washing was found. The only available facility for hand drying was non disposable cotton towel; this was found during $78.7 \%$ of the period of observation.

\subsection{Doctors' Hand Washing Practices}

Out of 86 doctors, 15 (17.4\%) washed hands before patients contact, while $55(64.0 \%)$ washed hands after patients contact. Table 1 shows doctors' hand washing practices before and after carrying out simple procedures. In both wards, hand washing rates were higher after carrying out simple procedures than before $(\mathrm{p}=0.00)$. During the period of observation, $80(93.0 \%)$ doctors used hand gloves. Out of these, $10(12.5 \%)$ washed their hands before wearing the gloves, while $62(77.5 \%)$ washed hands after removing the gloves. Seventy-one (88.8\%) doctors removed their gloves after use on each patient.

\subsection{Nurses' Hand Washing Practices}

Table 2 shows nurses hand washing practices before and after carrying out simple procedures. Nurses generally washed hands more frequently after the procedures than before $(p=0.00)$. Sixty-one $(95.3 \%)$ nurses used hand gloves during the period of observation. 
Table 1. Doctors hand washing practices before and after procedures

\begin{tabular}{llll}
\hline Procedures & Number of doctors & $\begin{array}{l}\text { Washed hands } \\
\text { before procedures }\end{array}$ & $\begin{array}{l}\text { Washed hands } \\
\text { after procedures }\end{array}$ \\
\hline EBT & $5(5.8)$ & $0(0)$ & $5(100)$ \\
Vene puncture & $66(76.4)$ & $15(22.7)$ & $40(60.6)$ \\
Lumber puncture & $23(26.7)$ & $13(56.5)$ & $20(87)$ \\
Urethral catheterization & $8(9.3)$ & $3(37.5)$ & $8(100)$ \\
\hline
\end{tabular}

Table 2. Nurses hand washing practices, before and after procedures

\begin{tabular}{llll}
\hline Procedures & Number of nurses & $\begin{array}{l}\text { Washed hands } \\
\text { before procedures }\end{array}$ & $\begin{array}{l}\text { Washed hands } \\
\text { after procedures }\end{array}$ \\
\hline NG tube feeding & $63(98.4)$ & $18(28.6)$ & $40(63.5)$ \\
Drug administration & $61(95.3)$ & $9(14.8)$ & $24(39.4)$ \\
Changed bed linens & $51(79.7)$ & $3(5.9)$ & $45(88.2)$ \\
Wound dressing & $32(50)$ & $5(15.6)$ & $27(84.4)$ \\
Taking of vital signs & $61(95.3)$ & $1(1.3)$ & $12(19.7)$ \\
\hline
\end{tabular}

Out of these, $9(14.8 \%)$ washed their hands before wearing the gloves, while $39(63.9 \%)$ washed their hands after removing the gloves. Forty-seven (77.0\%) nurses removed their gloves after use on each patient.

One hundred and eight $(72.0 \%)$ doctors and nurses dried their hands with non disposable common cotton towels, $28(18.7 \%)$ used personal hand kerchiefs to dry their hands and $14(9.3 \%)$ dried their hands on their ward coats. Only $6(4.0 \%)$ health workers washed their hands on entering the children's emergency ward and NICU, while $112(74.7 \%)$ washed hands before leaving the emergency room and NICU.

Generally, for both doctors and nurses, there was no significant relationship between time of day and hand washing practices $(\mathrm{p}=0.36)$.

\section{DISCUSSION}

Patients in the emergency room and NICU are critically ill and are subject to multiple hand contacts from a variety of staff. Busy places like these critical wards also provide the health workers with very frequent hand washing opportunities. The World Health Organization (WHO) hand hygiene guideline for health care specified that a hand hygiene action be performed whenever an opportunity for hand hygiene exists. WHO (2009). Failure to do this puts the patients at a great risk of nosocomial infections. It was therefore very disturbing to note that materials for hand washing such as soap and water were only available during a quarter of the period of observation.

The complete absence of any other hand hygiene agent during this period, coupled with the infrequent availability of soap and water will promote the transmission of pathogenic organisms from one patient to the other via the hands of health workers. Additionally, this situation will not promote the development of good hand washing/ hygiene practices by health workers. Two previous studies done in Nigeria and Eritrea also cited unavailability of soap and water as constraints to hand washing amongst health workers. (Alex-Hart and Opara, 2011; Samuel et al., 2005).

The study showed that health workers are most likely to wash hands after patient care. The WHO's guideline on hand hygiene specified that hand hygiene must be observed in 5 moments in health care settings. These are before patient contact, before aseptic task, after body fluids exposure risk, after patient contact and after contact with patients' surrounding. WHO (2009). In this study; hand washing was almost none existent amongst doctors and nurses before patient contact and before simple procedures. Even an aseptic procedure like lumbar puncture was not preceded by a very high rate of hand washing. Health workers probably assumed that wearing of gloves removed the need for hand washing. Other authors have expressed concern about the substitution of glove use for hand washing as this could promote the spread of nosocomial infections. (Larson and Killien, 1982; Doebbeling et al., 1988).

Hand washing rates dramatically improved to greater than $50 \%$ after patient contact and after simple procedures. This shows that health workers were primarily concerned with protecting themselves from acquiring pathogenic organisms from their patients; rather than trying to prevent the occurrence and spread of nosocomial infections amongst patients admitted under their care. This attitude of self preservation has been reported by other studies. (Lankford et al., 2003; Pittet, 2003). These findings also suggest that health workers 
in this hospital are probably not aware of the guidelines of infection control in health care settings. Hand washing education programmes that give specific considerations to the sequence of steps in patient care may address this problem.

The CDC (2005) hand hygiene guideline specified that hands should be washed before and after donning gloves. It was observed that though more than four fifth of the health workers used gloves at the appropriate times, only $12.5 \%$ of the doctors and $14.8 \%$ of nurses washed hands before donning the gloves. A previous study had identified the use of hand gloves as one of the factors responsible for low hand washing rates amongst health workers. Pittet (2001). Gloves reduce hand contamination by $70-80 \%$ and prevent patients and health care personnel from infection, but they do not eliminate the need for hand hygiene. CDC (2005). Some authors have also proven that wearing gloves does not always protect the hands of health workers from being colonized by some microorganisms. (Doebbeling et al., 1988; Pessoa-Silva et al., 2004) Meaning that health workers should also wash their hands after removing the gloves, this was complied with by 63.9 and $77.5 \%$ of the nurses and doctors (respectively) in this present study. In line with the CDC (2005) recommendation health workers in this study changed gloves between patients very frequently.

Effective hand drying is as important as proper hand washing in the hand hygiene process. This is because damp hands spread 1,000 times more micro-organisms than dry hands. Gould (1994). Common cotton towels were the only hand drying agent available in the wards throughout the period of observation. Not unexpectedly therefore, more than half $(72.0 \%)$ of the health workers were observed to dry their hands with it, while few others used personnel handkerchiefs $(18.7 \%)$ and their ward coats $(9.3 \%)$. This should be discouraged as it will result in further contamination of the hands of the health workers and encourage the spread of pathogenic organisms from one patient to the order. Ansari et al. (1991). The hospital should rather provide disposable paper towels which are the favoured hand drying agent. Gould (1994).

The study further revealed that whilst only $4 \%$ of the health workers washed their hands upon entering the emergency and neonatal intensive care wards, $74.7 \%$ washed their hands before leaving the wards. These finding also suggests a self-preservation attitude. In a previous self reported study carried out amongst health workers in the same hospital, only $25.2 \%$ reported washing their hands before leaving the wards. Alex-Hart and Opara (2011). This shows that self reports and actual observations are not always the same. Pritchard et al., (1996). The difference between these two studies, however could be that whilst the previous study was carried out amongst health workers from different departments, this present study was done only in critical sections of Paediatric care, where there is a high risk of contamination of health workers hands due to a high turnover of critically ill patients who mostly require resuscitative procedures. No significant relationship was found between health workers hand washing practices and the time of day. This contrasts with the findings in a previous study where hand washing rate was higher in the night time compared to the day time. Suchitra and Lakshmidevi (2006). The differences between these two studies could be that in the previous study, health workers were busier in the day time compared to the night time and this may have affected their hand washing practices. In this present study, the hand washing practices among the health workers was so poor that the time of day had no significant effect on it.

\section{CONCLUSION}

In conclusion, hand washing rates were very much lower amongst doctors and nurses before patient contact and simple procedures than after. Hand washing education programmes that give specific considerations to the sequence of steps in patient care may address the low hand washing rates before patient contact and before simple procedures. There is also need for provision of hand hygiene facilities within easy reach of HCWs.

\section{ACKNOWLEDGEMENT}

We would like to thank the Staff of Children Emergency and Neonatal Intensive Care Wards of University of Port Harcourt Teaching Hospital, Port Harcourt for working tireless to save the lives of children.

\section{REFERENCE}

Alex-Hart, B.A. and P.I. Opara, 2011. Handwashing practices amongst health workers in a teaching hospital. Am. J. Infect. Dis., 7: 8-15. DOI: 10.3844/ajidsp.2011.8.15

Akpaka, C.C., 2014. Best practices for hand hygiene compliance by health care providers in the inpatient setting. Am. J. Infect. Dis., 10: 84-87. DOI: 10.3844/ajidsp.2014.84.87 
Ansari, S.A., S.V. Springthorpe, S.A. Sattar, W. Tostowaryk and G.A. Wells., 1991. Comparison of cloth, paper and warm air in eliminating viruses and bacteria from washed hands. Am. J. Infect. Control, 19: 243-249. DOI: $10.1016 / \mathrm{S} 0196-6553$ (05)80256-1

Boyce, J.M., 2008. Hand hygiene compliance monitoring: Current perspective from USA. J. Hospital Infect., 70: 2-7. DOI: 10.1016/S01956701(08)60003-1

CDC, 2005. Hand hygiene guidelines. Fact Sheet, Centers for Disease Control.

Doebbeling, B.N., M.A. Pfaller, A.K. Houston and R.P. Wenzel, 1988. Removal of nosocomial pathogens from the contaminated glove. Implications of glove reuse and handwashing. Annals Internal Med., 109: 394-398. DOI: 10.7326/0003-4819-109-5-394

Faria, S., L. Sodano, A. Gjata, M. Dauri and A.F. Sabato et al., 2007. The first prevalence survey of nosocomial infections in the University Hospital Centre "Mother Teresa of Tirana", Abania. J. Hospital Infect., 65: 244-250. DOI: 10.1016/j.jhin.2006.11.007

Gosling, R., R. Mbatia, A. Savage, J.A. Mulligan and H. Reyburn., 2003. Prevalence of hospital-acquired infections in a tertiary referral hospital in Northern Tanzania. Annals Tropical Med. Parasitol., 97: 6973. PMID: 12662424

Gould, D., 1994. The significance of hand drying in the prevention of infection. Nurs. Times, 90: 33-35. PMID: 7800517

Jroundi, I., I. Khoudri, A. Azzouzi, A.A. Zeggwagh and N.F. Benbrahim et al., 2007. Prevalence of hospitalacquired infection in a Moroccan University hospital. Am. J. Infect. Control, 35: 412-416. DOI: 10.1016/j.ajic.2006.06.010

Kallel, H., M. Bahoul, H. Ksibi, H. Dammak and H. Chelly et al., 2005. Prevalence of hospital acquired infections in a Tunisian hospital. J. Hospital Infect., 59: 343-347. DOI: 10.1016/j.jhin.2004.09.015

Lankford, M.G., T.R. Zembower, W.E. Ttrck, D.M. Hacek and G.A. Noskin et al., 2003. Influence of role models and hospital design on hand hygiene of healthcare workers. Emerg. Infect. Dis., 9: 217-223. DOI: 10.3201/eid0902.020249

Larson, E.L., 1995. APIC guidelines for hand washing and hand antisepsis in health care settings. Am. J. Infect. Control, 23: 251-269. DOI: 10.1016/01966553(95)90070-5
Larson, E. and M. Killien., 1982. Factors influencing hand washing behavior of patient care personnel. Am. J. Infect. Control, 10: 93-99. PMID: 6922685

Pessoa-Silva, C.L., S. Dharan, S. Hugonnet, S. Touveneau and K. Posfay-Barbe et al., 2004. Dynamics of bacterial hand contamination during routine neonatal care. Infect. Control Hospital Epidemiol., 25: 192-197. DOI: 10.1086/502376

Pittet, D., A. Simon, S. Hugonnet, C.L. Pessoa-Silva and V. Sauvan et al., 2004. Hand hygiene among physicians: Performance, beliefs and perceptions. Annals Internal Med., 141: 1-8. DOI: 10.7326/00034819-141-1-200407060-00008

Pittet, D., P. Mourouga and T.V. Perneger, 1999. Compliance with handwashing in a teaching hospital. Infection control program. Annals Internal Med., 130: 126-13. DOI: 10.7326/0003-4819-130-2199901190-00006

Pittet, D., 2003. Improving compliance with hand hygiene.

Pittet, D., 2001. Improving adherence to hand hygiene practice: A multidisciplinary approach. Emerg. Infect. Dis., 7: 234-238. PMID: 11294714

Pritchard, R.C. and R.F. Raper, 1996. Doctors and handwashing: Instilling Semmelweiss' message. Med. J. Australia, 164: 389-390. PMID: 8609844

Samuel, R., A.M. Almedom, G. Hagos, S. Albin and A. Mutungi, 2005. Promotion of hand washing as a measure of quality of care and prevention of hospital acquired infections in Eritrea: The Keren study. African Health Sci., 5: 4-13. PMID: 15843125

Suchitra, J.B. and N. Lakshmidevi., 2006. Hand washing compliance-is it a reality? Online J. Health Allied Sci.

Vincent, J.L., 2003. Nosocomial infections in adult intensive care units. Lancet, 361: 2068-2077. DOI: 10.1016/S0140-6736(03)13644-6

WHO, 2005. World alliance for patients safety. World Health Organization.

WHO, 2009. Guidelines on hand hygiene in health care. First global patient safety challenge clean care is safer care. World Health Organization. 